\section{Beware the laboratory report: discrepancy in variant classification on reproductive carrier screening}

To the Editor: Carrier screening in the reproductive setting is designed to screen individuals without a clinical phenotype or family history to provide a risk assessment for genetic disorders. The number of conditions included on current screening panels range from 3 to over 200, with the majority of conditions following autosomal-recessive inheritance. In recent years several X-linked conditions have also been added, such as Duchenne muscular dystrophy (DMD) and Becker muscular dystrophy (BMD). Although carrier screening for DMD and BMD is not specifically recommended by any professional society, including the American College of Medical Genetics and Genomics (ACMG) and the American College for Obstetricians and Gynecologists, it is present on many laboratory panels. We present a prenatal screen in which a laboratory interpretation, had it not undergone further investigation, could have affected clinical outcome.

A 32-year-old G2P1001 Caucasian woman at 14 weeks gestation presented for genetic counseling with carrier screening results that reported her to be a carrier for BMD with a likely pathogenic variant. This variant, c.7850A $>\mathrm{G}$, is also known as NM_004006.2:c.8219A > G, p.D2740G in Human Genome Variation Society nomenclature. The laboratory reported that the variant was classified according to $\mathrm{ACMG}$ guidelines ${ }^{1}$ and had been previously observed in two other individuals with BMD. They reported a 50\% risk of having an affected pregnancy, as cell-free DNA screening had predicted a male fetus.

However, our own review of this variant showed inconsistencies with its classification. We contacted the original laboratory that first identified the variant and it was clarified that the two individuals reported in the literature were actually the same person: a 31-year-old man with a clinical diagnosis of BMD. Although this supports evidence of pathogenicity, as the patient's phenotype was highly specific for a disease with a single genetic etiology, functional clinical studies had not been completed. Additional information on this individual's phenotype was not available and it is presumed that he was lost to follow-up. No other case reports or studies on the variant's clinical implications could be found in the literature, muscular dystrophy database, or clinical variant databases. Our patient also denied any personal or family history of muscular dystrophy or cardiomyopathy.
The variant reported is classified as a missense mutation, which is a relatively uncommon cause of DMD and BMD. ${ }^{2}$ This should have supported evidence of a benign impact, as it is a missense variant in a gene where truncating mutations are known to primarily cause disease. Additionally, two other individuals in the Exome Aggregation Consortium (version 0.3.1; data released on 13 March 2016) have been noted with this variant, which suggests that it may be a rare variant in Europeans rather than a private variant only observed in those with BMD.

Given the uncertainty surrounding the variant, our patient declined prenatal diagnosis and elected to continue the pregnancy. Family studies were pursued, but provided limited clinical utility as no male relatives were identified with the variant. Our patient had an uneventful delivery at $392 / 7$ weeks gestation and testing for the NM_004006.2:c.8219A > G variant was pursued following delivery and confirmed in the male infant. The infant underwent serum creatine kinase testing and results were reported to be within normal limits. Therefore, despite the variant, this child is felt to be unaffected since creatine kinase levels are uniformly elevated in individuals with DMD or BMD at birth. ${ }^{3}$

If ACMG guidelines had been used, this variant should have been classified as a variant of unknown significance and thus not reported. It remains unclear how the reporting laboratory determined the classification of "likely pathogenic." Even if the reporting laboratory was correct in its assessment for the variant to be likely pathogenic, it is important to acknowledge that such classification would not have confirmed the variant to be disease causing. Using the $90 \%$ threshold suggested by ACMG for a variant to be labeled as likely pathogenic, there is still a $10 \%$ chance that it may be later reclassified. While many laboratories review available evidence and reclassify variants, they typically do so at intervals of 6 months to 1 year. In a pediatric or adult setting, care is often based on physical findings and a reclassification may not pose a large dilemma. In contrast, an improperly classified variant in prenatal care could have irreversible consequences as these families often have to make decisions on pregnancy management within a limited timeframe. Although prenatal diagnosis could be pursued in such situations, it would only identify the presence or absence of a variant without clarifying its clinical significance.

Additionally, it should be noted that the current guidelines in practice for variant interpretation and classification are not specific to those identified in prenatal care. Published in 2015, ACMG guidelines recommend a five-tiered system for indicating variant pathogenicity based on multiple lines of evidence, including population data, disease databases, segregation data, scientific and medical literature, and in silico predictors. Although most clinical laboratories report using ACMG guidelines, variant classification discrepancies between laboratories have already been noted in many areas of genetic testing. However, to our knowledge, this is the first 
presentation noted of a variant discrepancy in reproductive carrier screening.

In the past, variants in the prenatal realm have mostly been limited to use of chromosomal microarray and whole-exome sequencing where an ultrasound abnormality or family history may have already been noted. With the majority of disorders on carrier screening, there is often no significant family history or abnormal ultrasound findings expected to guide care. Such uncertain results are likely to increase parental anxiety and ultimately may result in potentially unnecessary invasive testing or pregnancy termination, which we strongly believe is not the intended goal of such screening. It is prudent that specific guidelines be created to direct variant interpretation and management in prenatal care as current reliance on a laboratory's interpretation is a disservice to our patients.

\section{DISCLOSURE}

The authors declare no conflict of interest.

\section{ACKNOWLEDGMENTS}

We would also like to thank the family described in this letter for being willing to share their story. Research reported in this publication was supported by the National Institute of
Neurological Disorders and Stroke of the National Institutes of Health under award R01NS085238 (R.B.W. and D.M.D.).

Aarti Ramdaney, $M S^{1}$, Diane M. Dunn, $B S^{2}$, Robert B. Weiss, $P h D^{2}$ and Nancy C. Rose, $M D^{3}$

${ }^{1}$ Department of Obstetrics, Gynecology and Reproductive Sciences, University of Texas Medical School at Houston, Houston, Texas, USA; ${ }^{2}$ Department of Human Genetics, University of Utah, Salt Lake City, Utah, USA; ${ }^{3}$ Department of Obstetrics and Gynecology, University of Utah, Salt Lake City, Utah, USA. Correspondence: Aarti Ramdaney (Aarti.Ramdaney@uth.tmc.edu)

\section{REFERENCES}

1. Richards S, Aziz N, Bale S, et al. Standards and guidelines for the interpretation of sequence variants: a joint consensus recommendation of the American College of Medical Genetics and Genomics and the Association for Molecular Pathology. Genet Med. 2015; 17:405-423.

2. Vulin A, Wein N, Strandjord DM, et al. The ZZ domain of dystrophin in DMD: making sense of missense mutations. Hum Mutat. 2014;35: 257-64.

3. Mendell JR, Shilling C, Leslie ND, et al. Evidence-based path to newborn screening for Duchenne muscular dystrophy. Ann Neurol. 2012;71: 304-13.

Advance online publication 26 October 2017. doi:10.1038/gim.2017.174 